\title{
Comparative Genomics Revealed Genetic Diversity and Species/Strain-Level Differences in Carbohydrate Metabolism of Three Probiotic Bifidobacterial Species
}

\author{
Toshitaka Odamaki, Ayako Horigome, Hirosuke Sugahara, Nanami Hashikura, \\ Junichi Minami, Jin-zhong Xiao, and Fumiaki Abe \\ Food Science and Technology Institute, Morinaga Milk Industry Co., Ltd., No. 1-83, 5-Chome Higashihara, Zama, Kanagawa, Japan \\ Correspondence should be addressed to Toshitaka Odamaki; t-odamak@morinagamilk.co.jp
}

Received 30 December 2014; Revised 4 May 2015; Accepted 26 May 2015

Academic Editor: John Parkinson

Copyright ( $) 2015$ Toshitaka Odamaki et al. This is an open access article distributed under the Creative Commons Attribution License, which permits unrestricted use, distribution, and reproduction in any medium, provided the original work is properly cited.

\begin{abstract}
Strains of Bifidobacterium longum, Bifidobacterium breve, and Bifidobacterium animalis are widely used as probiotics in the food industry. Although numerous studies have revealed the properties and functionality of these strains, it is uncertain whether these characteristics are species common or strain specific. To address this issue, we performed a comparative genomic analysis of 49 strains belonging to these three bifidobacterial species to describe their genetic diversity and to evaluate species-level differences. There were 166 common clusters between strains of B. breve and B. longum, whereas there were nine common clusters between strains of B. animalis and B. longum and four common clusters between strains of B. animalis and B. breve. Further analysis focused on carbohydrate metabolism revealed the existence of certain strain-dependent genes, such as those encoding enzymes for host glycan utilisation or certain membrane transporters, and many genes commonly distributed at the species level, as was previously reported in studies with limited strains. As B. longum and B. breve are human-residential bifidobacteria (HRB), whereas B. animalis is a non-HRB species, several of the differences in these species' gene distributions might be the result of their adaptations to the nutrient environment. This information may aid both in selecting probiotic candidates and in understanding their potential function as probiotics.
\end{abstract}

\section{Background}

The genus Bifidobacterium has been classified into 48 taxa, including 39 species and nine subspecies [1], three of which (Bifidobacterium longum, Bifidobacterium breve, and Bifidobacterium animalis) are commonly applied as bifidobacterial probiotics in the food industry. Many studies have been performed on the functionality of probiotic bifidobacteria in the host, and the properties and functionality of probiotics have generally been thought to be strain dependent [2, $3]$. However, previous reports have indicated that certain characteristics, such as the ability to generate vitamins [4], organic acids [5], and antimicrobial components $[6,7]$ as well as tolerance to stress $[8,9]$, are species dependent, although there is a degree of variation among strains. In several cases, it is unclear whether certain characteristics are species common or strain specific.

Recent work has provided genomic information for all bifidobacterial species/subspecies $[1,10]$. However, because the number of available genomic sequences from each species was limited in these studies, it is difficult to distinguish whether a number of unique genes are species or strain specific.

To further elucidate bifidobacterial genetic diversity and to evaluate species-level differences, we performed comparative genomic analyses of 49 strains belonging to three bifidobacterial species, or B. longum, B. breve, and B. animalis, which are commonly applied as probiotics in the food industry. The use of a large set of genome sequences based on a relatively large number of strains of each species allowed the 
identification of pan-genome structures and the elucidation of differences in core genome structures among these species. In the present report, given that bifidobacteria have been reported to utilise many unique metabolic pathways for sugar fermentation [11-19], additional analysis was focused on the carbohydrate transport/metabolism of each species/strain.

\section{Materials and Methods}

2.1. Bacterial Strains and Cultivation Conditions. The bacterial strains used in this study and general information about these strains are listed in Table 1 . The bifidobacterial strains were obtained from stock cultures maintained in the Morinaga Culture Collection (MCC; Morinaga Milk Industry Co., Ltd., Zama, Japan) and the American Type Culture Collection (ATCC, VA). Each strain was cultivated in Difco Lactobacilli MRS (Becton Dickinson, NJ) supplemented with 0.05\% Lcysteine. $\mathrm{HCl}$ (Kanto Chemical, Tokyo, Japan) at $37^{\circ} \mathrm{C}$ for $16 \mathrm{~h}$ under anaerobic conditions before DNA extraction. The microorganisms were collected by centrifugation, washed once with sterile saline, resuspended in an equivalent volume of sterile saline, and used as seed cultures for fermentation studies.

2.2. Assimilation of Human Milk Oligosaccharides. Modified MRS was prepared by removing glucose from the original components and was supplemented with LNT (Dextra Laboratories Ltd., Reading, UK) at a final concentration of $2 \%$. An aliquot of seed culture from each bifidobacterial strain was then inoculated into $200 \mu \mathrm{L}$ of the modified MRS, with a final concentration of $1 \%$. Growth of each bifidobacterial strain was measured by absorbance at OD600 after cultivation under anaerobic conditions at $37^{\circ} \mathrm{C}$ for $24 \mathrm{~h}$ and $48 \mathrm{~h}$. Experiments were performed in triplicate.

2.3. Genome Sequencing and Bioinformatics Analyses. Genomic DNA was extracted using the DNeasy Blood \& Tissue Kit (Qiagen, Valencia, CA) according to the manufacturer's protocol. The preparation of genomic libraries was performed with $1 \mathrm{ng}$ of genomic DNA using the Nextera XT DNA Sample Preparation Kit (Illumina Inc., CA) according to the manufacturer's instructions. After PCR amplification and cleanup, the fragment size distribution of the tagmented DNA was analysed using an Agilent 2100 Bioanalyzer and the High Sensitivity DNA Analysis Kit (Agilent Technologies, Santa Clara, CA). The libraries were sequenced using a MiSeq Personal Sequencing System and the MiSeq Reagent Kit v2 (500 cycles) (Illumina Inc.). Quality trimming and de novo assembly of the raw paired-end reads were performed using the CLC Genomics Workbench (v 6.0) software package (CLC bio, Aarhus, Denmark) with default settings, except for contig length (minimum contig length $=2,000 \mathrm{bp}$ ). Seventeen genomes that were sequenced de novo in this study were automatically annotated using the NCBI Prokaryotic Genome Annotation Pipeline (PGAP) 2.0 and were manually checked as part of the process of genome submission to GenBank. Our sequencing efforts resulted in multiple contigs (Table 1). The datasets for the genome annotations for the other 32 strains published previously were retrieved from the FTP server of NCBI [20].

2.4. Pan-Genome Analysis. For all of the 49 bifidobacterial strains included in this study, a pan-genome calculation was performed using the PGAP [21]. The ORF content of each genome was organised into functional gene clusters using the gene family (GF) method. Under the GF method, the total protein sequences of each strain were mixed together, with each gene being marked as a strain identifier. BLASTALL searches were performed among the mixed protein sequences, and the filtered BLAST results were clustered using the Markov Cluster algorithm [22], which has been widely used in other studies on prokaryotic genomes and in programs designed to search for orthologues among multiple strains. For each gene pair in a given cluster, the global match region was no less than $50 \%$ of the longer gene protein sequence, and the identity was also no less than $50 \%$. The minimum score value and $E$-value applied in BLAST were 50 and 1e-8. Pan-genome profile analysis, genetic variation analysis of functional genes, and function enrichment analysis of gene clusters were then performed. KEGG Orthology (KO) numbers were assigned by the KEGG Automatic Annotation Server (KAAS) using the bidirectional best-hit method [23].

2.5. Phylogenetic Trees. Pan-genome-based phylogenetic trees were generated according to a gene distance matrix, which was calculated based on genes that were absent or present in each strain [21], using UPGMA algorithms in PHYLIP. The sequences of 16S rRNA genes were aligned using the ClustalW alignment tool [24] with default parameters, and phylogenetic trees were constructed using UPGMA algorithms in MEGA6.06 [25]. Bootstrap values were calculated using 500 bootstrap replicates. The supertree was built using FigTree v1.4.0.

2.6. Nucleotide Sequence Accession Numbers. The de novo sequence and annotation data reported herein have been deposited in GenBank under the accession numbers AVQA00000000-AVQE00000000 and AWFK00000000AWFV00000000.

\section{Results and Discussion}

3.1. Phylogenetic Trees for Each Bifidobacterial Species. Based on the pan-genome profiles and 16S rRNA gene sequences, phylogenetic trees were constructed for each species, using Gardnerella vaginalis ATCC 14018 as an outgroup species. Each phylogenetic tree was divided into two clusters. However, the classification of subspecies of certain strains based on the genotypes of the strains was contradictory to that previously defined based on phenotypes (Table 1, Supplementary files 1-3 in Supplementary Material available online at http://dx.doi.org/10.1155/2015/567809). B. animalis subsp. animalis ATCC 27536 and ATCC 27674 fell into the clade with the type strain of $B$. animalis subsp. lactis, whereas B. longum subsp. longum JDM 301 and B. longum subsp. infantis $157 \mathrm{~F}$ fell into the clades with the type strains of B. longum 


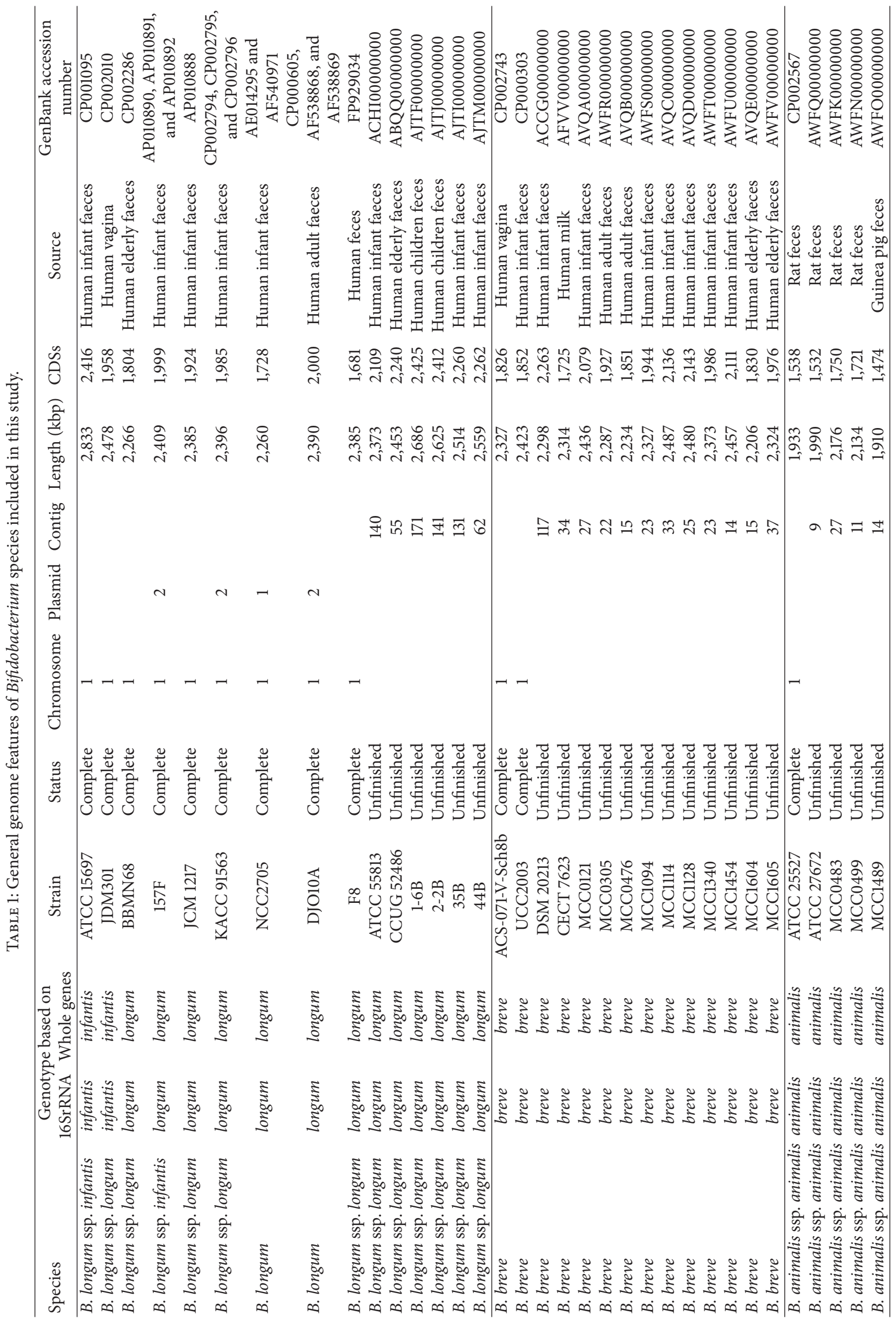




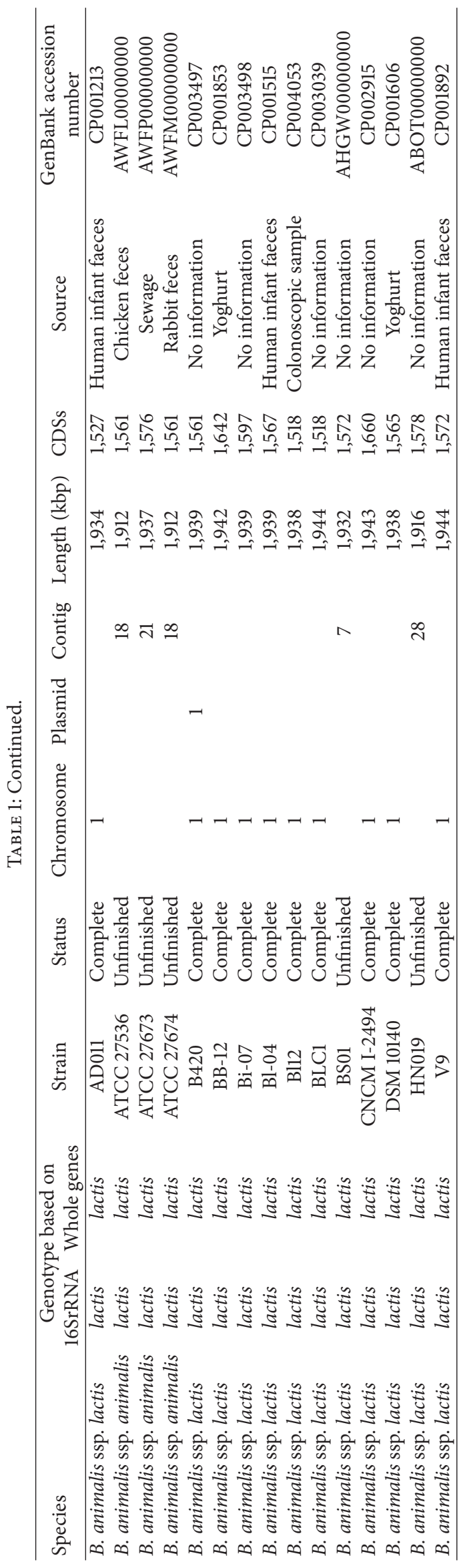




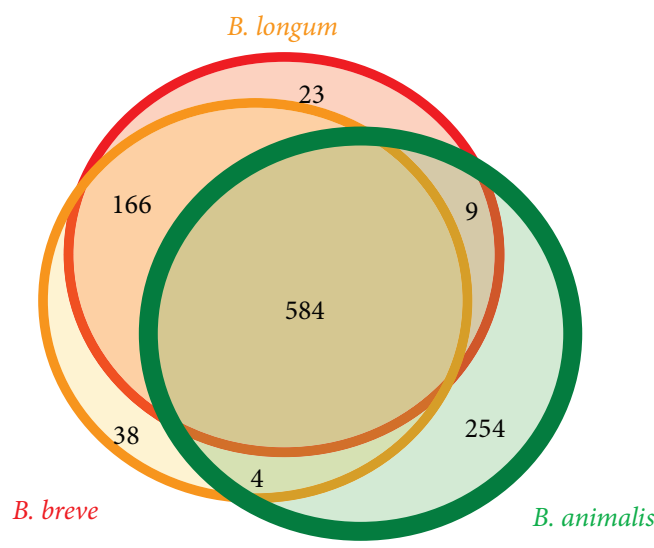

FIGURE 1: Venn diagram of the homologous clusters shared among the core genes. The surfaces are approximately proportional to the number of genes.

subsp. infantis and B. longum subsp. longum, respectively. In this study, we adopted subspecies of these strains based on the phylogenetic trees for further analyses.

3.2. Core and Pan-Genome Structures. After clustering the functional genes for each species, respective totals of 5,471, 4,053, and 2,833 clusters and 966, 1,221, and 1,092 core clusters were obtained for B. longum, B. breve, and B. animalis (Supplementary file 4). These numbers are similar to those reported previously [11, 26, 27]. However, we identified a wider variety of genomes in B. longum compared with a previous report [11]. This result may suggest that $B$. longum has an open pan-genome; that is, B. longum may exhibit a robust ability to import new genes, allowing it to adapt to each ecological niche over its long history of evolution. In the current study, to reveal differences among species, a total of 90,442 genes from the 49 strains were jointly clustered. These genes were divided into 8,818 homologous clusters, and there were 584 common clusters among all of the bifidobacterial strains (Figure 1). Additional analysis revealed that 404 of these clusters were commonly clustered with $G$. vaginalis ATCC 14018 as an outgroup species. Therefore, the number of specific gene clusters among the three bifidobacterial species was estimated to be less than 180. There were 166 common clusters between strains of $B$. breve and B. longum, whereas there were nine common clusters between strains of $B$. animalis and $B$. longum and four common clusters between strains of $B$. animalis and B. breve (Figure 1 ).

3.3. Metabolism of Host Glycans. Bifidobacteria have been reported to employ many unique metabolic pathways for sugar fermentation to enable them to utilise diverse carbohydrates in the intestine that are not utilised by their hosts [11-19]. Figure 2 shows the distributions of genes involved in the metabolism of host glycans, such as human milk oligosaccharides (HMOs), mucin, and N-glycans.

3.3.1. HMOs. A $43 \mathrm{~kb}$ cluster in the genome of B. longum subsp. infantis ATCC 15697 has been reported to be one of the most characteristic gene clusters for the utilisation of HMOs [28].

Seven homologous genes encoding extracellular solutebinding proteins predicted to bind oligosaccharides (SBP family 1) were found in only two strains of B. longum subsp. infantis, whereas homologues of the major facilitator superfamily (Blon_2331, 2332) were found in all strains except for B. animalis subsp. lactis AD011. Two of four gene homologues encoding glycoside hydrolases (alpha-galactosidase and beta$\mathrm{N}$-acetylhexosaminidase) were found in strains of $B$. breve as well as B. longum subsp. infantis. Fucosidase (Blon_2336) and sialidase (Blon_2348) homologues were found only in $B$. longum subsp. infantis. However, certain strains of $B$. breve possessed other gene clusters related to fucose and sialic acid incorporation (Figure 2).

Regarding the core structure of HMOs, three of the four predominant HMOs (lacto-N-fucopentaose I (LNFP I), lacto-N-difucohexaose I (LNDFH I), and lacto-N-tetraose (LNT)) exhibit the LNT structure. Therefore, the enzyme required for the utilisation of LNT is crucial in the utilisation of HMOs and the colonisation of the infant intestine. Certain strains belonging to B. bifidum, B. longum subsp. longum, B. longum subsp. infantis, and B. breve have been reported to act as LNT consumers [29, 30]. The last two species incorporate intact LNT via an unidentified transporter and then hydrolyse it intracellularly using LNT $\beta$ 1,3-galactosidase (Blon_2016) [31]. In our study, using type strains, we confirmed the ability of B. longum subsp. longum, $B$. longum subsp. infantis, and $B$. breve, but not $B$. animalis subsp. animalis and B. animalis subsp. lactis, in the utilisation of LNT (Supplementary file 5). Unexpectedly, Blon_2016 homologues were also present in B. animalis strains. Based on phylogenetic analysis, Yoshida et al. [31] discovered that there is a close homologue of this gene (amino acid identity $>95 \%$ ) that falls into a clade of strains of infant gut-related species (i.e., B. breve, B. longum subsp. infantis, and $B$. longum subsp. longum), whereas the gene in B. animalis was observed to be distant from this clade. These results imply the existence of different substrate specificity for LNT $\beta-1,3$ galactosidase, although further investigations are necessary to examine this issue. B. bifidum and certain strains of $B$. longum subsp. longum produce a secretory enzyme, LNBase, that hydrolyses LNT into lacto- $N$-biose (LNB) and lactose $[32,33]$. In the present study, homologous genes encoding LNBase (BLLJ_1506) and the chaperone for this enzyme (BLLJ_1505) were found in two strains of B. longum subsp. longum. The LNBase of B. longum subsp. longum has been reported to be able to hydrolyse the GlcNAc $\beta 1-3 \mathrm{Gal}$ linkage in LNT, LNFP I, and sialyllacto-N-tetraose. These results imply the existence of different mechanisms for the utilisation of LNT decorated by fucose and sialic acid. B. bifidum might digest LNT after releasing its own fucosidase and sialidase, whereas B. longum subsp. longum lacks these enzymes but can directly degrade decorated LNT.

Subsequently, the liberated LNB is imported into the cells via the galacto-N-biose (GNB)/LNB transporter for further degradation [34]. The disaccharide is then phosphorolysed by a GNB/LNB phosphorylase to produce Gal-1-P and GlcNAc 


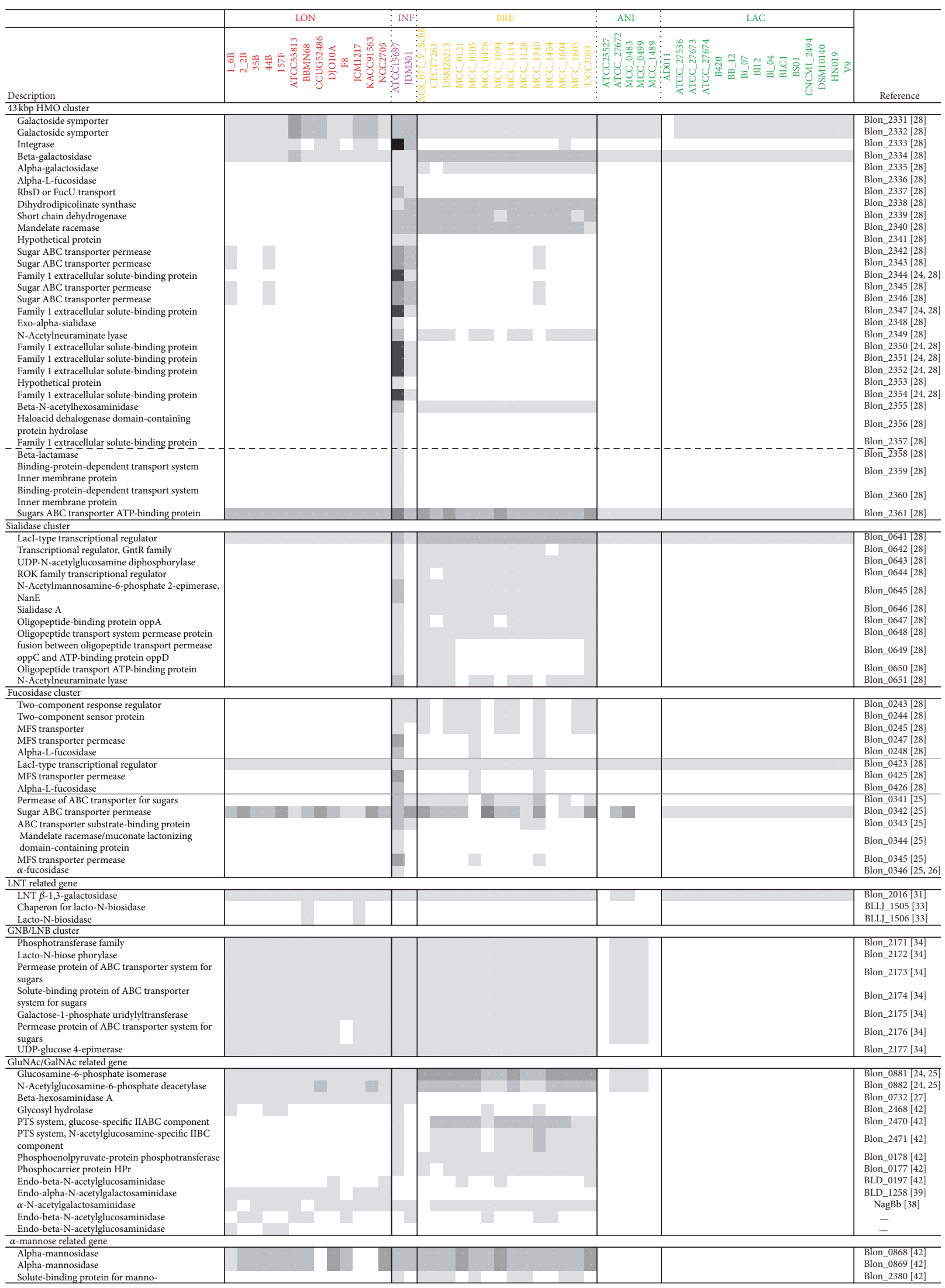

FIGURE 2: Distribution of genes involved in the metabolism of host glycans, such as human milk oligosaccharides, mucin, and N-glycans. The abbreviations used for species/subspecies are as follows: INF, B. longum subsp. infantis; LON, B. longum subsp. longum; BRE, B. breve; ANI, B. animalis subsp. animalis; LAC, B. animalis subsp. lactis. 


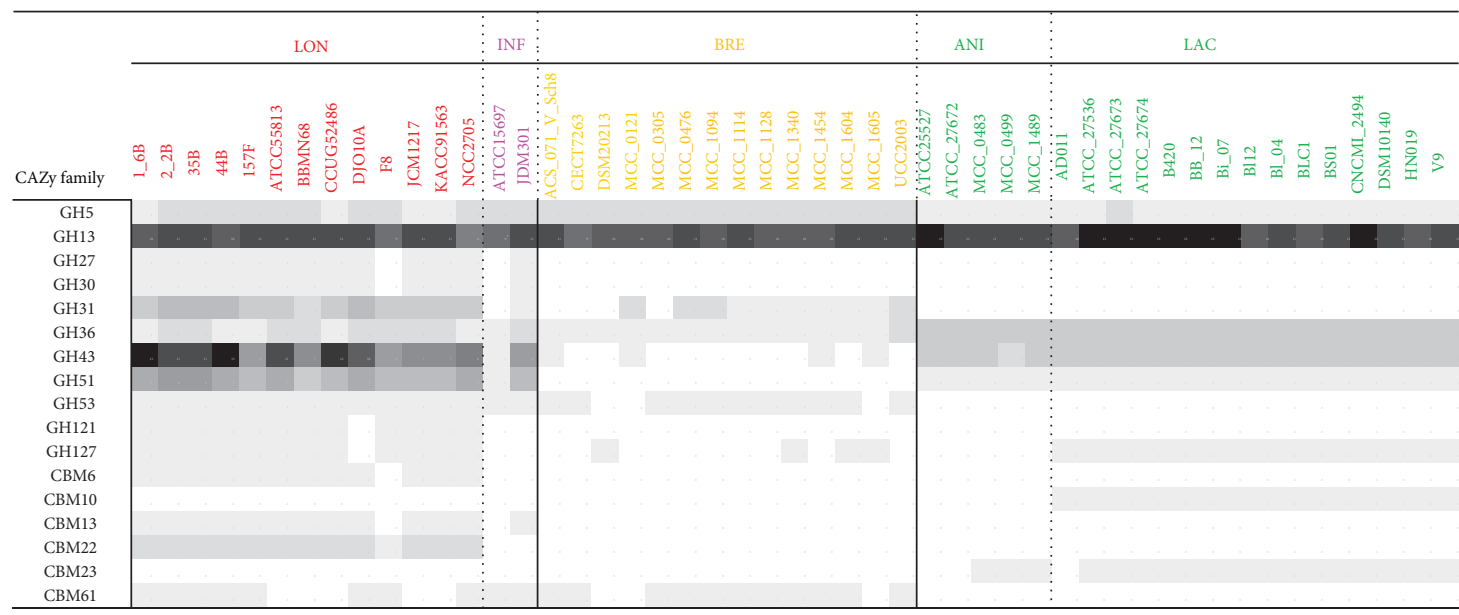

FIgURE 3: Distribution of genes involved in the metabolism of plant-derived sugars predicted by the CAZy database. The abbreviations used for species/subspecies are as follows: INF, B. longum subsp. infantis; LON, B. longum subsp. longum; BRE, B. breve; ANI, B. animalis subsp. animalis; LAC, B. animalis subsp. lactis.

and is further metabolised [34]. In the current study, a sevengene operon involved in this pathway was observed in nearly all strains of $B$. longum and $B$. breve and in three strains of $B$. animalis subsp. animalis. This distribution is in complete accord with results regarding in vitro LNB utilisation [35]. A previous report revealing a strong ability to grow in human milk also supports these claims [36].

3.3.2. Mucin and N-Glycans. Another substrate for this metabolic pathway is GNB, which is a core structure of gastrointestinal mucin. Mucins are extensively O-glycosylated proteins and are thought to serve as a potential carbon source for gut microbiota. Studies have revealed that the main core structures of gastric/duodenal and intestinal mucins are core1, 2-type and core-3-type O-glycans, respectively [37, 38]. A homologous gene encoding $\alpha-N$-acetylgalactosaminidase, acting on core-3-type O-glycan (NagBb) [39], was observed in 2 strains of $B$. longum subsp. infantis, 11 strains of $B$. longum subsp. longum, and 13 strains of $B$. breve in the present study. In contrast, a gene encoding endo- $\alpha$ $\mathrm{N}$-acetylgalactosaminidase, which is predicted to release GNB-containing glycans from core-1-type O-glycan mucin (BLD_1258) [40], was observed only in B. longum subsp. longum. In addition, the terminal ends of glycoconjugates in the suckling gut have been reported to predominantly consist of sialic acid, whereas in the adult, they predominantly consist of fucose $[41,42]$. In our study, nearly all strains of $B$. longum subsp. infantis and $B$. breve contained a gene encoding sialidase, which might be useful for this species to colonise the infant intestine.

Most strains of B. longum and B. breve, but not $B$. animalis strains, were found to possess genes involved in the pathway for the utilisation of N-acetylglucosamine (GlcNAc) and GalNAc, which are the major constituents of host glycans. Furthermore, gene homologues encoding endo-betaN-acetylglucosaminidase (BLD_0197) [43], which releases complex $\mathrm{N}$-glycans from human milk glycoproteins, and alpha-mannosidase (Blon_0868, 0869) were found in certain strains of these two species. These enzymes involved in the degradation of host glycans might play a role in the utilisation of intrinsic carbohydrate sources.

Bifidobacteria are generally residents of the intestines of animals, including warm-blooded mammals and social insects, and several bifidobacterial species are typical inhabitants of the human gut (designated human-residential bifidobacteria or HRB). All strains of B. longum and B. breve, which are typical $\mathrm{HRB}$, possess certain genes related to the pathway for the utilisation of LNT/LNB, which are core structures of type I oligosaccharides that are specific to human breast milk (Figure 2). Among HRB strains in our study, nearly all possessed gene operons involved in the GNB/LNB pathway, whereas genes upstream of HMO utilisation, such as fucosidase, sialidase, and LNBase, were species/strain dependent. These results suggest that each HRB strain might evolve to assimilate HMOs, for which hundreds of types of structures have been reported [44]. In contrast, $B$. animalis is a non-HRB species; although certain strains of $B$. animalis subsp. lactis have also been isolated from humans, they have been found in faecal samples but rarely in colon mucosal samples [27, 45]. In addition, previous reports have shown that $B$. animalis is a strictly monophyletic group, and the evolutionary distance between $B$. animalis and species of HRB was shown to be relatively far based on $16 \mathrm{~S}$ rRNA multigene alignments and comparative genomics $[11,13,27,46]$. Our results indicate that there are fewer homologues involved in the degradation of host glycan by $B$. animalis (Figure 2). These results suggest that the observed differences in the gene distribution might be the result of the adaptation of these strains to their residential environments.

\subsection{Carbohydrate-Active Enzymes for Plant-Derived Sugars.} Regarding extrinsic carbohydrates, a variety of resistant fibres, which can be dietary compounds, are delivered to the colon, where bifidobacteria reside. Based on CAZy classification, all of the strains possessed a large number of homologous genes encoding GH 13 family members (Figure 3), which has previously been reported as a characteristic feature of bifidobacterial genomes [47, 48]. 


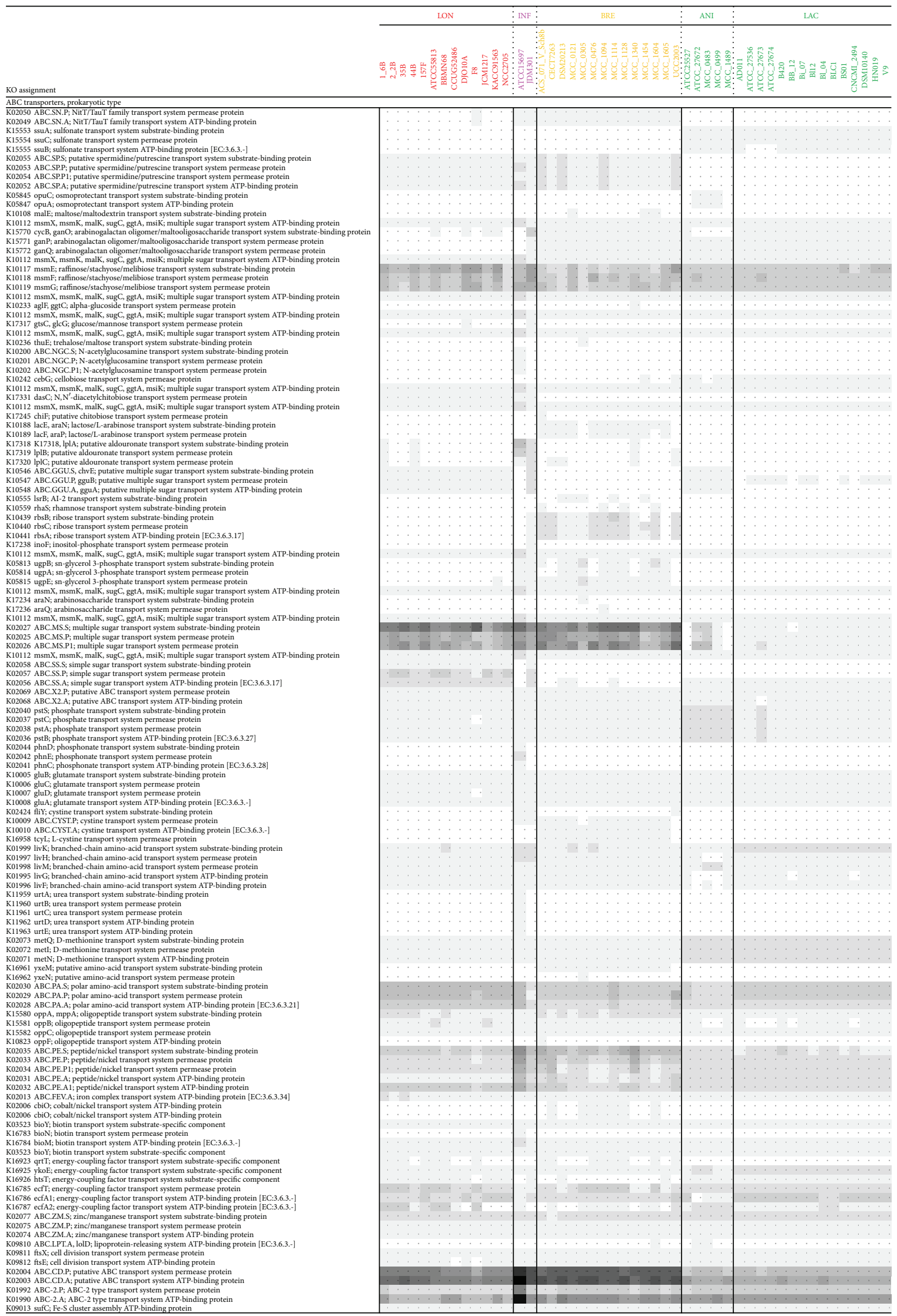

FIgURE 4: Continued. 


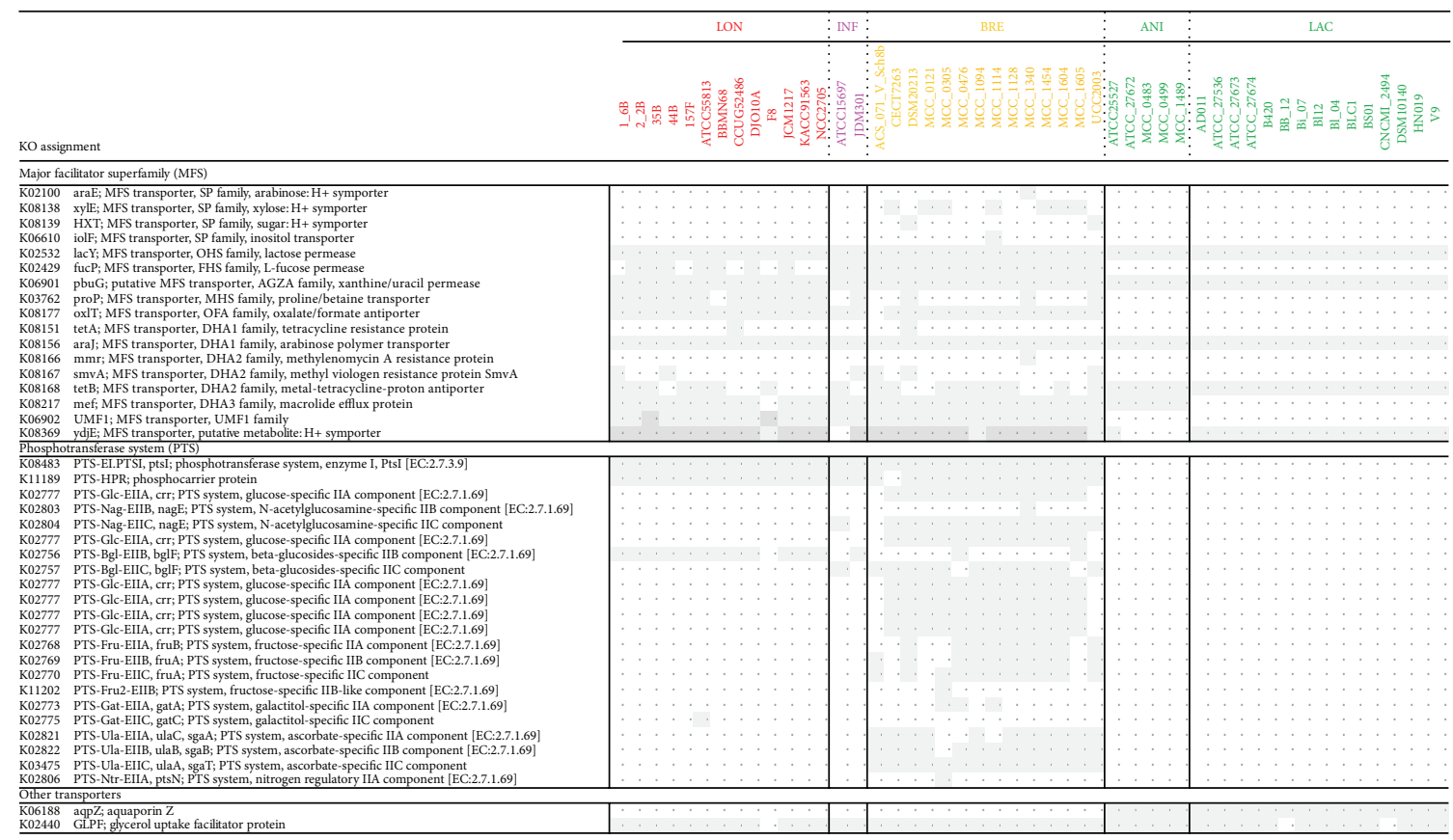

FIGURE 4: Distribution of genes encoding membrane transporters for carbohydrate. The abbreviations used for species/subspecies are as follows: INF, B. longum subsp. infantis; LON, B. longum subsp. longum; BRE, B. breve; ANI, B. animalis subsp. animalis; LAC, B. animalis subsp. lactis.

These enzymes are typical enzymes for the degradation of alpha-glucopyranose units such as pullulan, starch, and amylopectin. In particular, B. longum subsp. longum was shown to be genetically well equipped for the fermentation of plant-derived sugars (Figure 3), which are assumed to be not introduced into the infant gut before weaning. A large number of GH 43 and 51 family members, which are enzymes responsible for the degradation of arabinose/xylose units such as arabinofuranoside and xylan, were found to be specific to $B$. longum subsp. longum, with strain specificity for several of these genes. Taken together with the distribution of genes for host glycan utilisation, such a wide range of genes for carbohydrate utilisation provides an advantage for colonisation of the human intestine by B. longum subsp. longum. This result might explain why only $B$. longum subsp. longum is a predominant species in both the infant and the adult intestines [49].

3.5. Membrane Transporters for Carbohydrate. KO assignment indicated several differences in the distribution of carbohydrate transporters at the subspecies level. The subspecies that possessed the greatest number of transporter homologues was $B$. longum subsp. infantis $(83.5 \pm 2.1)$, including taxon-specific transporters for GlcNAc, phosphonate, and urea, whereas $B$. animalis subsp. lactis possessed the lowest number of these homologues (33.7 \pm 1.3$)$. All three species can assimilate fructose; however, only species of $B$. longum exhibited a set of high-affinity fructose-specific ABC transporters (K02056, K02057, and K02058), which have been reported to be involved in efficiently converting fructose to acetate under certain gut conditions [5]. B. longum subsp. longum lacked a homologous gene set for arabinogalactan transport. However, twelve strains of B. longum subsp. longum possessed a set of homologous genes encoding extracellular enzymes for the degradation of arabinogalactan (BLLJ_1840) [50]. B. breve also exhibited certain unique $\mathrm{ABC}$ transporters for lactose/Larabinose, ribose, and sn-glycerol 3-phosphate.

We found distinctive differences between the three species in terms of the distribution of genes related to the phosphotransferase system (PTS), which is known to be a multicomponent system specific for sugar uptake that operates in global carbon regulation in many bacteria [51]. Strains of B. longum and B. breve possessed three homologues, encoding phosphoenolpyruvate-protein kinase, phosphocarrier protein, and beta-glucoside-PTS, whereas none of the $B$. animalis strains exhibited these genes. Additionally, nearly all strains of $B$. breve possessed homologues of $\mathrm{N}$ acetylglucosamine, glucose, fructose, and ascorbate, which are involved in the PTS (Figure 4).

\section{Conclusions}

Certain strains belonging to the three bifidobacterial species targeted in this study have been reported to exert numerous beneficial effects on human health as probiotics. Probiotic functionality is generally thought to be strain dependent. In fact, our analysis confirmed that certain genes, such as those encoding membrane transporters and enzymes for host glycan utilisation, are strain dependent. However, our data also demonstrated that there are common characteristics in each species that may be important in light of the species' health-promoting effects on their hosts. Additionally, 
differences in characteristics might be a result of adaptation to the nutrient environments of each species (such as HRB versus non-HRB). Our results support previous observations based on the investigation of certain type strains of bifidobacterial species and enable the qualification of several of these characteristics as species common or strain specific $[33,39,50,52]$. Taken together with other characteristics, such as vitamin metabolism $[4,53]$, colonisation factors [54], and extracellular components $[55,56]$, we believe that these findings will help to predict the features of probiotic strains. However, further study is needed to evaluate other non-HRB and HRB bifidobacterial species to attain a better understanding of the characteristics of these bacteria as well as the mechanisms underlying their residence in the host intestine and their potential functions as probiotics.

\section{Conflict of Interests}

All authors are employees of Morinaga Milk Industry Co., Ltd., which has several probiotic products marketed worldwide.

\section{Acknowledgments}

The authors wish to thank Kensuke Suzuki and Keiichiro Nishihama (Illumina Inc.) for technical support.

\section{References}

[1] G. A. Lugli, C. Milani, F. Turroni et al., "Investigation of the evolutionary development of the genus Bifidobacterium by comparative genomics," Applied and Environmental Microbiology, vol. 80, no. 20, pp. 6383-6394, 2014.

[2] M. Meijerink, J. M. Wells, N. Taverne et al., "Immunomodulatory effects of potential probiotics in a mouse peanut sensitization model," FEMS Immunology and Medical Microbiology, vol. 65, no. 3, pp. 488-496, 2012.

[3] Y. Liu, N. Y. Fatheree, N. Mangalat, and J. M. Rhoads, "Humanderived probiotic Lactobacillus reuteri strains differentially reduce intestinal inflammation," The American Journal of Physiology-Gastrointestinal and Liver Physiology, vol. 299, no. 5, pp. G1087-G1096, 2010.

[4] M. R. D’Aimmo, P. Mattarelli, B. Biavati, N. G. Carlsson, and T. Andlid, "The potential of bifidobacteria as a source of natural folate," Journal of Applied Microbiology, vol. 112, no. 5, pp. 975984, 2012.

[5] S. Fukuda, H. Toh, K. Hase et al., "Bifidobacteria can protect from enteropathogenic infection through production of acetate," Nature, vol. 469, no. 7331, pp. 543-547, 2011.

[6] N. Pandey, R. K. Malik, J. K. Kaushik, and G. Singroha, "Gassericin A: a circular bacteriocin produced by Lactic acid bacteria Lactobacillus gasseri," World Journal of Microbiology and Biotechnology, vol. 29, no. 11, pp. 1977-1987, 2013.

[7] M. R. Islam, J.-I. Nagao, T. Zendo, and K. Sonomoto, "Antimicrobial mechanism of lantibiotics," Biochemical Society Transactions, vol. 40, no. 6, pp. 1528-1533, 2012.

[8] L. Masco, C. Crockaert, K. van Hoorde, J. Swings, and G. Huys, "In vitro assessment of the gastrointestinal transit tolerance of taxonomic reference strains from human origin and probiotic product isolates of Bifidobacterium," Journal of Dairy Science, vol. 90, no. 8, pp. 3572-3578, 2007.
[9] E. Rosberg-Cody, A. Liavonchanka, C. Göbel et al., "Myosincross-reactive antigen (MCRA) protein from Bifidobacterium breve is a FAD-dependent fatty acid hydratase which has a function in stress protection," BMC Biochemistry, vol. 12, no. 1, article 9, 2011.

[10] C. Milani, G. A. Lugli, S. Duranti et al., "Genomic encyclopedia of type strains of the genus Bifidobacterium," Applied and Environmental Microbiology, vol. 80, no. 20, pp. 6290-6302, 2014.

[11] F. Bottacini, D. Medini, A. Pavesi et al., "Comparative genomics of the genus Bifidobacterium," Microbiology, vol. 156, part 11, pp. 3243-3254, 2010.

[12] F. Turroni, D. van Sinderen, and M. Ventura, "Genomics and ecological overview of the genus Bifidobacterium," International Journal of Food Microbiology, vol. 149, no. 1, pp. 37-44, 2011.

[13] O. Lukjancenko, D. W. Ussery, and T. M. Wassenaar, "Comparative genomics of Bifidobacterium, Lactobacillus and related probiotic genera," Microbial Ecology, vol. 63, no. 3, pp. 651-673, 2012.

[14] M. A. Schell, M. Karmirantzou, B. Snel et al., "The genome sequence of Bifidobacterium longum reflects its adaptation to the human gastrointestinal tract," Proceedings of the National Academy of Sciences of the United States of America, vol. 99, no. 22, pp. 14422-14427, 2002.

[15] P. Hao, H. Zheng, Y. Yu et al., "Complete sequencing and pangenomic analysis of Lactobacillus delbrueckii subsp. bulgaricus reveal its genetic basis for industrial yogurt production," PLoS ONE, vol. 6, no. 1, Article ID e15964, 2011.

[16] J.-H. Kim, H. J. An, D. Garrido, J. B. German, C. B. Lebrilla, and D. A. Mills, "Proteomic analysis of bifidobacterium longum subsp. infantis reveals the metabolic insight on consumption of prebiotics and host glycans," PLoS ONE, vol. 8, no. 2, Article ID e57535, 2013.

[17] D. Garrido, J. H. Kim, J. B. German, H. E. Raybould, and D. A. Mills, "Oligosaccharide binding proteins from bifidobacterium longum subsp. infantis reveal a preference for host glycans," PLoS ONE, vol. 6, no. 3, Article ID e17315, 2011.

[18] D. A. Sela, D. Garrido, L. Lerno et al., “Bifidobacterium longum subsp. infantis ATCC $15697 \alpha$-fucosidases are active on fucosylated human milk oligosaccharides," Applied and Environmental Microbiology, vol. 78, no. 3, pp. 795-803, 2012.

[19] D. Garrido, S. Ruiz-Moyano, and D. A. Mills, "Release and utilization of $\mathrm{N}$-acetyl-d-glucosamine from human milk oligosaccharides by Bifidobacterium longum subsp. infantis," Anaerobe, vol. 18, no. 4, pp. 430-435, 2012.

[20] ftp://ftp.ncbi.nlm.nih.gov/genomes/.

[21] Y. Zhao, J. Wu, J. Yang, S. Sun, J. Xiao, and J. Yu, "PGAP: pangenomes analysis pipeline," Bioinformatics, vol. 28, no. 3, Article ID btr655, pp. 416-418, 2012.

[22] A. J. Enright, S. Van Dongen, and C. A. Ouzounis, "An efficient algorithm for large-scale detection of protein families," Nucleic Acids Research, vol. 30, no. 7, pp. 1575-1584, 2002.

[23] Y. Moriya, M. Itoh, S. Okuda, A. C. Yoshizawa, and M. Kanehisa, "KAAS: an automatic genome annotation and pathway reconstruction server," Nucleic Acids Research, vol. 35, no. 2, pp. W182-W185, 2007.

[24] J. D. Thompson, D. G. Higgins, and T. J. Gibson, "CLUSTAL W: improving the sensitivity of progressive multiple sequence alignment through sequence weighting, position-specific gap penalties and weight matrix choice," Nucleic Acids Research, vol. 22, no. 22, pp. 4673-4680, 1994. 
[25] K. Tamura, G. Stecher, D. Peterson, A. Filipski, and S. Kumar, "MEGA6: molecular evolutionary genetics analysis version 6.0," Molecular Biology and Evolution, vol. 30, no. 12, pp. 2725-2729, 2013.

[26] F. Bottacini, M. O. C. Motherway, J. Kuczynski et al., "Comparative genomics of the Bifidobacterium breve taxon," $B M C$ Genomics, vol. 15, no. 1, article 170, 2014.

[27] C. Milani, S. Duranti, G. A. Lugli et al., "Comparative genomics of Bifidobacterium animalis subsp. lactis reveals a strict monophyletic bifidobacterial taxon," Applied and Environmental Microbiology, vol. 79, no. 14, pp. 4304-4315, 2013.

[28] D. A. Sela, J. Chapman, A. Adeuya et al., "The genome sequence of Bifidobacterium longum subsp. infantis reveals adaptations for milk utilization within the infant microbiome," Proceedings of the National Academy of Sciences of the United States of America, vol. 105, no. 48, pp. 18964-18969, 2008.

[29] S. Asakuma, E. Hatakeyama, T. Urashima et al., "Physiology of consumption of human milk oligosaccharides by infant gutassociated bifidobacteria," The Journal of Biological Chemistry, vol. 286, no. 40, pp. 34583-34592, 2011.

[30] R. E. Ward, M. Niñonuevo, D. A. Mills, C. B. Lebrilla, and J. B. German, "In vitro fermentability of human milk oligosaccharides by several strains of bifidobacteria," Molecular Nutrition and Food Research, vol. 51, no. 11, pp. 1398-1405, 2007.

[31] E. Yoshida, H. Sakurama, M. Kiyohara et al., "Bifidobacterium longum subsp. infantis uses two different $\beta$-galactosidases for selectively degrading type- 1 and type- 2 human milk oligosaccharides," Glycobiology, vol. 22, no. 3, pp. 361-368, 2012.

[32] J. Wada, T. Ando, M. Kiyohara et al., "Bifidobacterium bifidum lacto-N-biosidase, a critical enzyme for the degradation of human milk oligosaccharides with a type 1 structure," Applied and Environmental Microbiology, vol. 74, no. 13, pp. 3996-4004, 2008.

[33] H. Sakurama, M. Kiyohara, J. Wada et al., "Lacto- $N$-biosidase encoded by a novel gene of Bifidobacterium longum subspecies longum shows unique substrate specificity and requires a designated chaperone for its active expression," The Journal of Biological Chemistry, vol. 288, no. 35, pp. 25194-25206, 2013.

[34] M. Nishimoto and M. Kitaoka, "Identification of $N$-acetylhexosamine 1-kinase in the complete lacto- $N$-biose I/galacto- $N$ biose metabolic pathway in Bifidobacterium longum," Applied and Environmental Microbiology, vol. 73, no. 20, pp. 6444-6449, 2007.

[35] J.-Z. Xiao, S. Takahashi, M. Nishimoto et al., "Distribution of in vitro fermentation ability of lacto-N-biose I, a major building block of human milk oligosaccharides, in bifidobacterial strains," Applied and Environmental Microbiology, vol. 76, no. 1, pp. 54-59, 2010.

[36] F. Turroni, E. Foroni, F. Serafini et al., "Ability of Bifidobacterium breve to grow on different types of milk: exploring the metabolism of milk through genome analysis," Applied and Environmental Microbiology, vol. 77, no. 20, pp. 7408-7417, 2011.

[37] J. R. Gum Jr., J. W. Hicks, N. W. Toribara, B. Siddiki, and Y. S. Kim, "Molecular cloning of human intestinal mucin (MUC2) cDNA. Identification of the amino terminus and overall sequence similarity to prepro-von Willebrand factor," The Journal of Biological Chemistry, vol. 269, no. 4, pp. 24402446, 1994.

[38] J. M. H. Larsson, H. Karlsson, H. Sjövall, and G. C. Hansson, "A complex, but uniform O-glycosylation of the human MUC2 mucin from colonic biopsies analyzed by nanoLC/MS"," Glycobiology, vol. 19, no. 7, pp. 756-766, 2009.
[39] M. Kiyohara, T. Nakatomi, S. Kurihara et al., " $\alpha$-N-acetylgalactosaminidase from infant-associated bifidobacteria belonging to novel glycoside hydrolase family 129 is implicated in alternative mucin degradation pathway," The Journal of Biological Chemistry, vol. 287, no. 1, pp. 693-700, 2012.

[40] K. Fujita, F. Oura, N. Nagamine et al., "Identification and molecular cloning of a novel glycoside hydrolase family of core 1 type $O$-glycan-specific endo- $\alpha-N$-acetylgalactosaminidase from Bifidobacterium longum," Journal of Biological Chemistry, vol. 280, no. 45, pp. 37415-37422, 2005.

[41] M. C. Biol, A. Martin, M. Richard, and P. Louisot, "Developmental changes in intestinal glycosyl-transferase activities," Pediatric Research, vol. 22, no. 3, pp. 250-256, 1987.

[42] D. Dai, N. N. Nanthkumar, D. S. Newburg, and W. A. Walker, "Role of oligosaccharides and glycoconjugates in intestinal host defense," Journal of Pediatric Gastroenterology and Nutrition, vol. 30, no. 2, pp. S23-S33, 2000.

[43] D. Garrido, C. Nwosu, S. Ruiz-Moyano et al., "Endo- $\beta-N-$ acetylglucosaminidases from infant gut-associated bifidobacteria release complex N-glycans from human milk glycoproteins," Molecular and Cellular Proteomics, vol. 11, no. 9, pp. 775-785, 2012.

[44] T. Urashima, S. Asakuma, F. Leo, K. Fukuda, M. Messer, and O. T. Oftedal, "The predominance of type I oligosaccharides is a feature specific to human breast milk," Advances in Nutrition, vol. 3, no. 3, pp. 473S-482S, 2012.

[45] F. Turroni, E. Foroni, P. Pizzetti et al., "Exploring the diversity of the bifidobacterial population in the human intestinal tract," Applied and Environmental Microbiology, vol. 75, no. 6, pp. 1534-1545, 2009.

[46] M. Ventura, C. Canchaya, A. del Casale et al., "Analysis of bifidobacterial evolution using a multilocus approach," International Journal of Systematic and Evolutionary Microbiology, vol. 56, no. 12, pp. 2783-2792, 2006.

[47] F. Bottacini, M. Ventura, D. Van Sinderen, and M. O. Motherway, "Diversity, ecology and intestinal function of bifidobacteria," Microbial Cell Factories, vol. 13, supplement 1, article S4, 2014.

[48] S. M. Ryan, G. F. Fitzgerald, and D. Van Sinderen, "Screening for and identification of starch-, amylopectin-, and pullulandegrading activities in bifidobacterial strains," Applied and Environmental Microbiology, vol. 72, no. 8, pp. 5289-5296, 2006.

[49] K. Yamamoto, Science of Lactic Acid Bacteria and Bifidobacteria, Japan Society for Lactic Acid Bacteria, 2010.

[50] K. Fujita, T. Sakaguchi, A. Sakamoto, M. Shimokawa, and K. Kitahara, "Bifidobacterium longum subsp. longum exo- $\beta$ 1,3-galactanase, an enzyme for the degradation of type II arabinogalactan," Applied and Environmental Microbiology, vol. 80, no. 15, pp. 4577-4584, 2014.

[51] R. Brückner and F. Titgemeyer, "Carbon catabolite repression in bacteria: choice of the carbon source and autoregulatory limitation of sugar utilization," FEMS Microbiology Letters, vol. 209, no. 2, pp. 141-148, 2002.

[52] D. Garrido, D. C. Dallas, and D. A. Mills, "Consumption of human milk glycoconjugates by infant-associated bifidobacteria: mechanisms and implications," Microbiology, vol. 159, part 4, pp. 649-664, 2013.

[53] R. G. Crittenden, N. R. Martinez, and M. J. Playne, "Synthesis and utilisation of folate by yoghurt starter cultures and probiotic bacteria," International Journal of Food Microbiology, vol. 80, no. 3, pp. 217-222, 2003. 
[54] M. O. Motherway, A. Zomer, S. C. Leahy et al., "Functional genome analysis of Bifidobacterium breve UCC2003 reveals type IVb tight adherence (Tad) pili as an essential and conserved host-colonization factor," Proceedings of the National Academy of Sciences of the United States of America, vol. 108, no. 27, pp. 11217-11222, 2011.

[55] J. A. Tamayo-Ramos, J. M. Sanz-Penella, M. J. Yebra, V. Monedero, and M. Haros, "Novel phytases from Bifidobacterium pseudocatenulatum ATCC 27919 and Bifidobacterium longum subsp. infantis ATCC 15697," Applied and Environmental Microbiology, vol. 78, no. 14, pp. 5013-5015, 2012.

[56] D. Ivanov, C. Emonet, F. Foata et al., "A serpin from the gut bacterium Bifidobacterium longum inhibits eukaryotic elastaselike serine proteases," The Journal of Biological Chemistry, vol. 281, no. 25, pp. 17246-17252, 2006. 

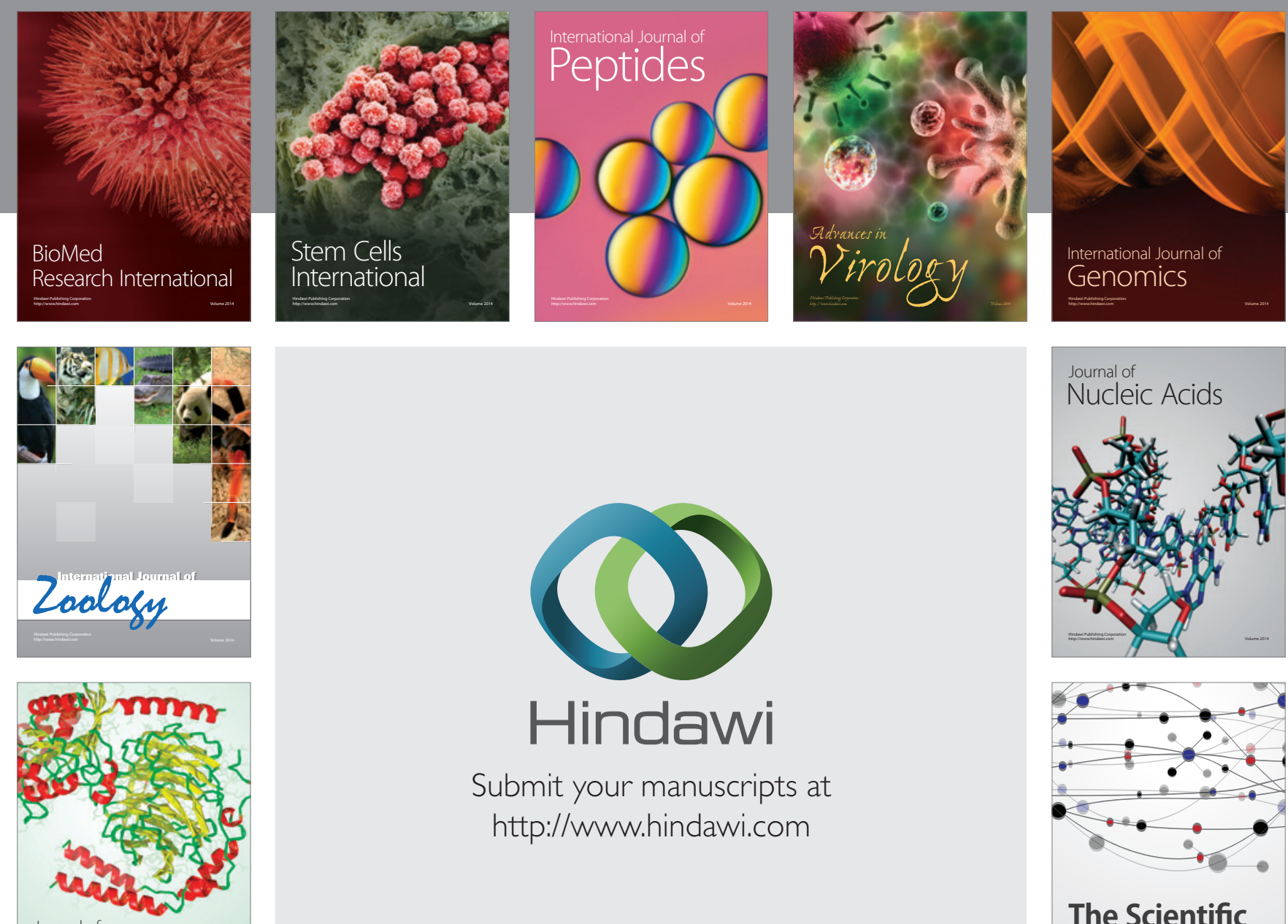

Submit your manuscripts at

http://www.hindawi.com

Journal of
Signal Transduction
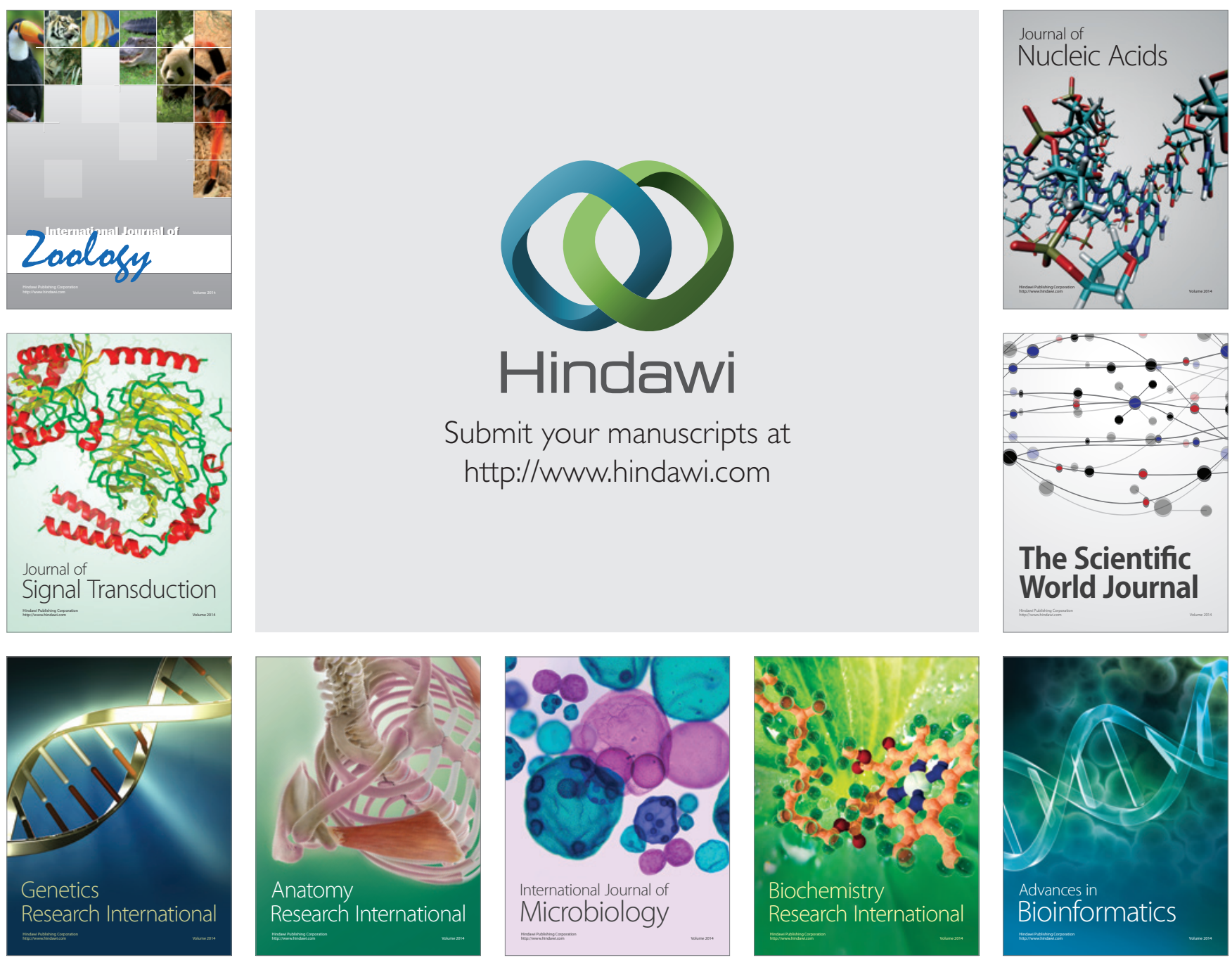

The Scientific World Journal
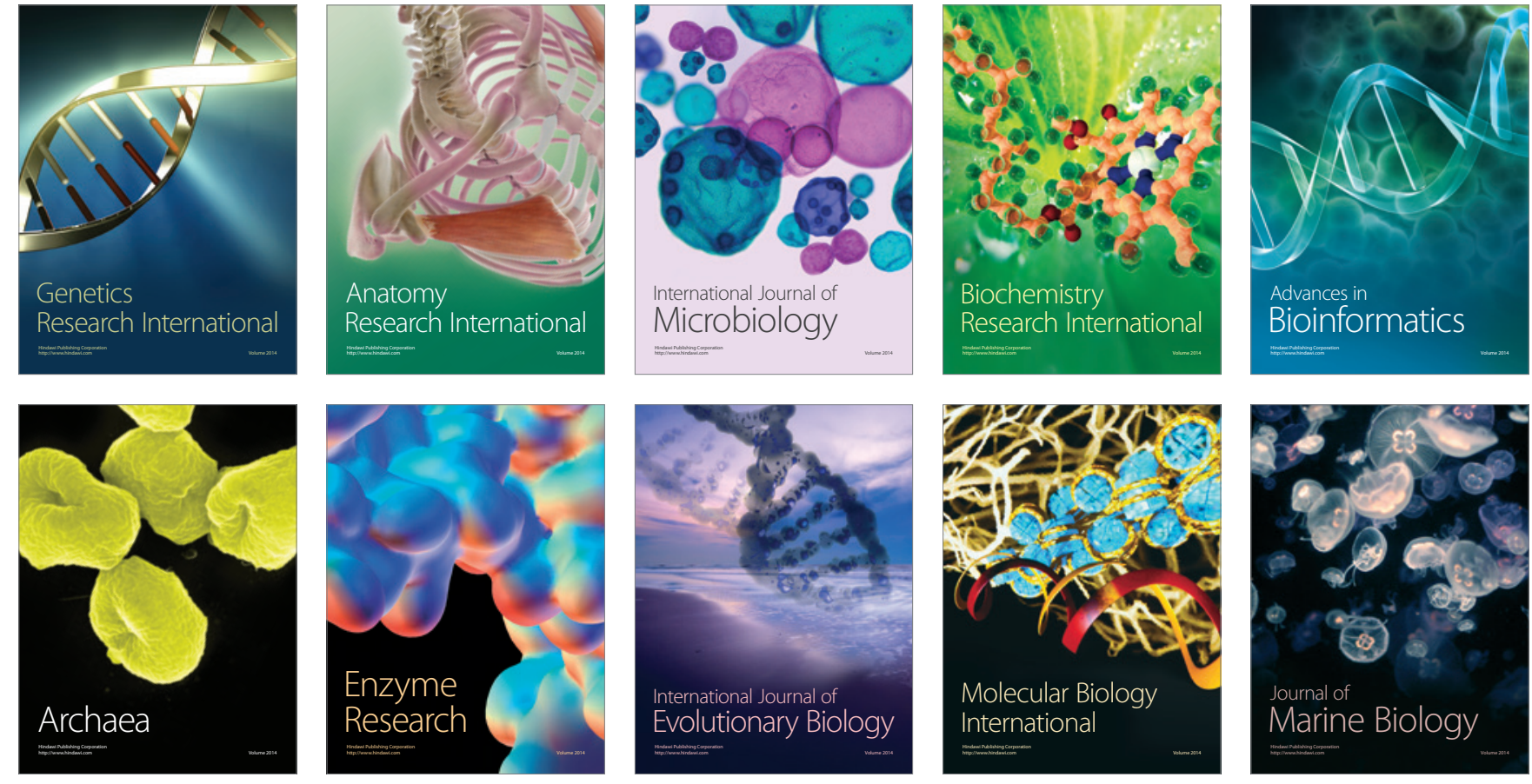